\title{
STRINGY ROBINSON-TRAUTMAN SOLUTIONS
}

\author{
R. Güven \\ Department of Mathematics, \\ Boğaziçi University \\ Bebek, İstanbul 80815, Turkey \\ and \\ E. Yörük \\ Department of Physics, \\ Boğaziçi University \\ Bebek, İstanbul 80815,Turkey
}

\begin{abstract}
A class of solutions of the low energy string theory in four dimensions is studied. This class admits a geodesic, shear-free null congruence which is non-twisting but in general diverging and the corresponding solutions in Einstein's theory form the Robinson-Trautman family together with a subset of the Kundt's class. The Robinson-Trautman conditions are found to be frame invariant in string theory. The Lorentz Chern-Simons three form of the stringy Robinson-Trautman solutions is shown to be always closed. The stringy generalizations of the vacuum Robinson-Trautman equation are obtained and three subclasses of solutions are identified. One of these subclasses exists, among all the dilatonic theories, only in Einstein's theory and in string theory. Several known solutions including the dilatonic black holes, the pp- waves, the stringy C-metric and certain solutions which correspond to exact conformal field theories are shown to be particular members of the stringy Robinson-Trautman family. Some new solutions which are static or asymptotically flat and radiating are also presented. The radiating solutions have a positive Bondi mass. One of these radiating solutions has the property that it settles down smoothly to a black hole state at late retarded times.
\end{abstract}

PACS numbers: 11.25.Mj, 04.70.Dy, 04.30.Nk 


\section{Introduction}

In general relativity Robinson-Trautman solutions [1] have proven to be an interesting laboratory for addressing certain issues of black holes, gravitational radiation and the asymptotic structure of space times. These solutions are singled out by requiring that the spacetime admits a shear-free, non-twisting, geodesic null congruence. In Einstein-Maxwell theory this requirement leads to a large class of algebraically special solutions which belong to various Petrov types and one can identify the Reissner-Nordström black holes, the charged C-metric as well as certain radiating solutions as particular members of the Robinson-Trautman family [2]. The explicit forms of all the Robinson-Trautman solutions are, however, not known and the relevance of some of these solutions to the black hole formation [3] as well as the structure of the Robinson-Trautman equations [4] are still problems of current interest.

In this paper we wish to study the Robinson-Trautman solutions of string theory in four dimensions. We shall look for the solutions of the low energy string theory which admit, in the Einstein frame, a shear-free, nontwisting, geodesic null congruence. We shall derive the stringy generalization of the Robinson-Trautman equation and examine the various subclasses of solutions. One of these subclasses has the feature that it distinguishes, among all the dilatonic theories, Einstein's theory and the string theory. This subclass exists only in these two theories. The whole family of stringy Robinson-Trautman solutions, however, turns out to be interesting for several other reasons. For example, we shall show that Robinson-Trautman conditions are frame invariant in string theory. Hence if a solution is of the Robinson-Trautman type in the Einstein frame, then it will also be a Robinson-Trautman solution in the string frame. It is also worth noting that these solutions do not admit in general any Killing vectors. Due to this property, the whole family cannot be generated from the vacuum solutions of Einstein's equations by Ehlers-Harrison type transformations [5]. Another attractive feature concerns the Lorentz Chern-Simons three-form. We shall prove that the Lorentz Chern-Simons three-form of the RobinsonTrautman family is always closed. This property is relevant to the higher order corrections and we shall see that the stringy Robinson-Trautman family contains, in fact, several exact solutions or the leading order representations of exact conformal field theories. One of the exact solutions which is of the Robinson-Trautman type is that of an electrically charged, extreme black 
hole [6], [7]. Another such solution can be identified by noting that, in contrast to Einstein-Maxwell theory and because of the presence of the dilaton, it is possible to switch off the divergence of the geodesic null congruence within the stringy Robinson-Trautman family. By specializing to this case and choosing the spacetime to be conformally flat, one can arrive at the special plane wave solution which is interpretable as a WZW model [8] and plane waves are also known to be exact solutions [9]. These two examples show that the Robinson-Trautman family has a non-empty intersection with the chiral null models [10]. We shall recover several other known solutions as particular members of the Robinson-Trautman family. It will be seen that the family contains the set of all charged dilatonic black holes [6], the pp-waves [11], the stringy C-metric [12], the static, spherically symmetric solutions of [13] as well as the solutions of [14] which correspond to exact conformal field theories. We shall also present explicitly some new solutions which are static or asymptotically flat and radiating. The radiating solutions that we shall study have a positive Bondi mass. One of these radiating solutions tends smoothly to the stringy black holes at late retarded times.

\section{Robinson-Trautman Form of the Fields}

Most of our discussions are based on the action

$$
S=\int d^{4} x \sqrt{-g}\left[R+2 \nabla_{\mu} \phi \nabla^{\mu} \phi-\tilde{\kappa}^{2} e^{-2 a \phi} F_{\mu \nu} F^{\mu \nu}\right],
$$

where $R$ is the Ricci scalar for the metric $g_{\mu \nu}$, the real scalar field $\phi$ is the dilaton, $F_{\mu \nu}=\partial_{\mu} A_{\nu}-\partial_{\nu} A_{\mu}$ is the spin-1 field strength, $\tilde{\kappa}$ is the coupling constant, $g=\operatorname{det}\left(g_{\mu \nu}\right)$ and $a$ is a real parameter. When $0 \leq a \leq 1$, this action describes the dilatonic gravity theories which interpolate between the standard Einstein-Maxwell theory $(a=0, \phi=$ const.) and the low-energy string theory $(a=1)$. The field equations that follow from (2.1) can be written as

$$
\begin{gathered}
d \star\left(e^{-2 a \phi} F\right)=0 \\
d \star d \phi-a \tilde{\kappa}^{2} e^{-2 a \phi} F \wedge \star F=0 \\
R_{\mu \nu}=-2 \nabla_{\mu} \phi \nabla_{\nu} \phi+2 \tilde{\kappa}^{2} e^{-2 a \phi}\left(F_{\mu \lambda} F_{\nu}^{\lambda}-\frac{1}{4} g_{\mu \nu} F_{\kappa \lambda} F^{\kappa \lambda}\right),
\end{gathered}
$$


where $F=\frac{1}{2} F_{\mu \nu} d x^{\mu} \wedge d x^{\nu}$ is the Maxwell two-form, $R_{\mu \nu}$ is the Ricci tensor and $\star$ denotes the Hodge dual. It can be checked that these equations are invariant under the duality transformations

$$
F \rightarrow e^{-2 a \phi} \star F, \quad \phi \rightarrow-\phi .
$$

We shall be primarily concerned with the $a=1$ case of (2.1). In identifying this case as the low-energy string action one assumes that the axion field is set equal to zero. The axion field strength, however, involves the spin-1 Chern-Simons three-form and for a proper identification, the field equations must be complemented with

$$
F \wedge F=0
$$

Since the gravitational part of (2.1) has the standard Einstein-Hilbert form, $g_{\mu \nu}$ is the Einstein metric and the $a=1$ specializations of (2.2)-(2.4) together with (2.6) are the string field equations in the Einstein frame. To pass to the string frame, one must introduce at $a=1$

$$
g_{\mu \nu}^{s}=e^{2 a \phi} g_{\mu \nu},
$$

and transform (2.1) so that the string metric $g_{\mu \nu}^{s}$ is the gravitational field variable.

Our first goal is to characterize the Robinson-Trautman solutions of the string field equations in the Einstein frame. For this purpose it will be convenient to employ the Newman-Penrose (NP) formalism [15] and work with a null tetrad $\left(l_{\mu}, n_{\mu}, m_{\mu}, \bar{m}_{\mu}\right)$. The null tetrad determines the metric as

$$
g_{\mu \nu}=2 l_{(\mu} n_{\nu)}-2 m_{(\mu} \bar{m}_{\nu)} .
$$

The NP form of the Cartan's equations of structure and the decomposition of the spacetime curvature are described in the Appendix. The spin-1 field, on the other hand, is represented by three complex scalars $\Phi_{0}, \Phi_{1}, \Phi_{2}$ and these may be defined by

$$
F+i \star F=-2 \Phi_{1}(l \wedge n-m \wedge \bar{m})-2 \Phi_{0} n \wedge \bar{m}+2 \Phi_{2} l \wedge m,
$$

where $l=l_{\mu} d x^{\mu}, n=n_{\mu} d x^{\mu}, m=m_{\mu} d x^{\mu}$ are the null basis one forms; $l$ and $n$ are real, $m$ is complex. Throughout the paper an overbar denotes 
complex conjugation. We shall choose $l^{\mu}$ to be tangent to a geodesic, shearfree, non-twisting null congruence. In terms of the NP spin coefficients, this means

$$
\kappa=\sigma=0, \quad \rho=\bar{\rho} .
$$

We shall also assume that $l^{\mu}$ is a null eigenvector of $F_{\mu \nu}$ :

$$
\Phi_{0}=0,
$$

and impose on the full energy momentum tensor the conditions

$$
\Phi_{01}=\Phi_{02}=0 .
$$

If one were to impose (2.10) and (2.11) in Einstein-Maxwell theory, the spacetime would be algebraically special:

$$
\Psi_{0}=\Psi_{1}=0,
$$

where $\Psi_{0}, \Psi_{1}$ are Weyl scalars (see Appendix) and (2.12) would follow from (2.11). What one is really dealing with would then be a particular case of the Goldberg-Sachs theorem [2]. Because the dilaton also contributes to the energy-momentum tensor, this is no longer the case in string theory. We shall require the stringy Robinson-Trautman family to share the algebraic character of its Einstein counterpart and impose (2.13) as an additional condition on the spacetime curvature. Hence the solutions which obey (2.10)-(2.13) will constitute the stringy Robinson-Trautman family.

The null vector $l^{\mu}$ is now an eigenvector of both $F_{\mu \nu}$ and the Weyl tensor and the tetrad gauge freedom is partially fixed. There is still the freedom of performing null rotations which preserve the direction of $l^{\mu}$ and these involve four real parameters. Using such a null rotation the complex connection one-form $\Gamma_{1}$ defined, in the Appendix, can be reduced to

$$
\Gamma_{1}=\rho m,
$$

and as a consequence of (2.12) and (2.13) one finds

$$
\Gamma_{1} \wedge d \Gamma_{1}=0 .
$$

The Frobenius theorem then allows one to introduce two arbitrary functions $P_{0}$ and $z$ such that

$$
\Gamma_{1}=\left(\rho / P_{0}\right) d \bar{z}
$$


and $P_{0}$ can always be choosen to be real. Then, according to (2.14) and (2.16), the complex leg of the null tetrad must have the form

$$
m=\frac{1}{P_{0}} d \bar{z} .
$$

Since (2.10) is assumed to hold, $l$ also satisfies

$$
l \wedge d l=0
$$

and the Frobenius theorem can be utilized once again together with a null rotation which preserves $(2.17)$ to set

$$
l=d u,
$$

where $u$ is an arbitrary real function. In order to determine the form of the final leg $n$, let us choose as our coordinate system $(u, r, z, \bar{z})$, where $r$ is an affine parameter along the null geodesic tangent to $l^{\mu}$. In such a coordinate system the most general $n$ can be written as

$$
n=d r+H_{0} d u+W d \bar{z}+\bar{W} d z
$$

where $H_{0}$ is a real function and $W$ is complex. Computing now all the NP spin coefficients for (2.17), (2.19) and (2.20) gives

$$
W^{\prime}=0, \quad W_{z}=\bar{W}_{\bar{z}}
$$

Here and in the sequel we use primes to denote differentiation with respect to $r$ whereas the other partial derivatives are denoted by subscripts: $W^{\prime}=$ $\partial W / \partial r, W_{z}=\partial W / \partial z, W_{u}=\partial W / \partial u$. It follows from the conditions (2.21) that one can reduce $(2.20)$ to the form

$$
n=d r+H d u
$$

by a coordinate transformation: $r \rightarrow r+f(u, z, \bar{z})$ without altering the forms of $l$ and $m$. When the last two terms of (2.20) are gauged away by setting $f_{z}=\bar{W}$, the coefficient of $d u$ transforms into a new function $H$ and $r$ is now the new coordinate. Hence there exists a coordinate system $(u, r, z, \bar{z})$ where one is left only with two real functions $P_{0}$ and $H$. In this coordinate system (2.10)-(2.13) are all satisfied by an arbitrary $H$ but $P_{0}$ must obey

$$
\left(\ln P_{0}\right)_{z}^{\prime}=0
$$


in order to fulfill $\Psi_{1}=0$. Thus the final outcome of the conditions (2.10)(2.13) can be written as

$$
\begin{aligned}
l & =d u, \\
n & =d r+H d u, \\
m & =\frac{R}{P} d \bar{z},
\end{aligned}
$$

where $H=H(u, r, z, \bar{z}), R=R(u, r), P=P(u, z, \bar{z})$ are real functions. The stringy Robinson-Trautman line element must therefore be of the form

$$
d s^{2}=2 d u d r+2 H d u^{2}-2 R^{2} \frac{d z d \bar{z}}{P^{2}} .
$$

The conditions (2.11) and (2.12) have also nontrivial implications on the dilaton through the field equation (2.4). The vanishing of the $\Phi_{01}$ component of the Ricci tensor requires $\phi^{\prime} \phi_{z}=0$ and $\Phi_{02}=0$ implies $\left|\phi_{z}\right|^{2}=0$. Hence the allowable dilaton fields are of the form

$$
\phi=\phi(u, r)
$$

and we now have the most general forms of $g_{\mu \nu}, \phi$ and $F_{\mu \nu}$ that belong to the Robinson-Trautman family. Notice that this family is defined for all dilatonic theories.

Let us next impose (2.6). For an arbitrary Maxwell field and in terms of the NP variables, (2.6) amounts to

$$
\Phi_{1}^{2}-\Phi_{0} \Phi_{2}=\bar{\Phi}_{1}^{2}-\bar{\Phi}_{0} \bar{\Phi}_{2}
$$

In our case $\Phi_{0}=0$ and therefore, $\Phi_{1}= \pm \bar{\Phi}_{1}$. Since the electric case: $\Phi_{1}=\bar{\Phi}_{1}$ and the magnetic case: $\Phi_{1}=-\bar{\Phi}_{1}$ can be mapped to one another by a duality transformation (2.5), we shall choose to work only with the magnetic solutions:

$$
\Phi_{1}=-\bar{\Phi}_{1}
$$

The problem is now to determine two real functions $R, \phi$ of two variables, a real function $P$ of three variables together with a real $H$, a purely imaginary $\Phi_{1}$ and a complex $\Phi_{2}$ where the last three functions can depend on all the 
coordinates. Before embarking into the differential equations which govern these functions three points are worth noticing:

First, if one compares the above results with those of the Einstein-Maxwell theory, one finds as the new feature of the stringy Robinson-Trautman metric, the presence of a general warp factor $R(u, r)$. As we shall see below this warp factor is coupled to the dilaton. Whenever $\phi=$ const., $R^{\prime \prime}=0$ and without any loss of generality one can take $R=r$ in Einstein-Maxwell theory. According to (2.3) it is, of course, not permissible to set $\phi=$ const. unless $a=0$ or $F \wedge \star F=0$. In Einstein's theory the metric (2.25) was previously encountered in perfect fluid solutions [16].

Secondly, suppose one chooses to work with the string metric $g_{\mu \nu}^{s}$ rather than the Einstein metric. The starting point of the foregoing analysis will then be the null tetrad of $g_{\mu \nu}^{s}$. Because of (2.7), this tetrad can be related to (2.24) by the conformal transformation

$$
l^{s}=e^{a \phi} l, \quad n^{s}=e^{a \phi} n, \quad m^{s}=e^{a \phi} m .
$$

It can be checked by computing the connection and the curvature of the new tetrad that, as long as $\phi=\phi(u, r)$, the conditions (2.10)-(2.13) will remain to hold in the string frame. Hence if a solution is of the Robinson-Trautman type in the Einstein frame, it will also be a Robinson-Trautman solution in the string frame.

Finally, let us consider the Lorentz Chern-Simons three-form in the Einstein frame. The Lorentz Chern-Simons three-form $\omega_{3 L}^{0}$ is known to satisfy

$$
d \omega_{3 L}^{0}=\Omega_{a b} \wedge \Omega^{a b}
$$

where $\Omega_{a b}$ are the curvature two-forms. In terms of the NP variables one finds that

$$
\star d \omega_{3 L}^{0}=4 i\left[3\left(\bar{\Psi}_{2}^{2}-\Psi_{2}^{2}\right)+\left(\bar{\Psi}_{0} \bar{\Psi}_{4}-\Psi_{0} \Psi_{4}\right)+4\left(\Psi_{1} \Psi_{3}-\bar{\Psi}_{1} \bar{\Psi}_{3}\right)\right] .
$$

When one specializes to the Robinson-Trautman metrics, $\Psi_{0}=\Psi_{1}=0$ and moreover, it turns out that $\Psi_{2}=\bar{\Psi}_{2}$ (see Appendix). Hence for any metric having the form $(2.25)$

$$
d \omega_{3 L}^{0}=0
$$

and consequently, the axion field which was taken to be zero in the leading order approximation, can be maintained to be zero even after including $\omega_{3 L}^{0}$ as a higher order correction. Notice that (2.25) is more general than the metrics which are known to enjoy this property [17]. 


\section{The Field Equations}

We are now in a position to consider the field equations which govern the Robinson-Trautman form of the fields. Among these Maxwell equations (2.2) are the simplest:

$$
\begin{gathered}
\left(R^{2} \Phi_{1}\right)^{\prime}=0, \\
\left(R \Phi_{2}\right)^{\prime}=0, \\
\Phi_{1 z}+a R \phi^{\prime}\left(\bar{\Phi}_{2} / P\right)=0, \\
\left(R^{2} \Phi_{1} / P^{2}\right)_{u}+R\left(\Phi_{2} / P\right)_{z}=0 .
\end{gathered}
$$

The first two equations can be readily integrated to give

$$
\Phi_{1}=\frac{c}{R^{2}}, \quad \Phi_{2}=\frac{h}{R}
$$

where $c=c(u, z, \bar{z}), h=h(u, z, \bar{z})$ and because (2.28) holds, $c=-\bar{c}$. Hence $\Phi_{1}$ and $\Phi_{2}$ depend on the coordinate $r$ only through the function $R(u, r)$. When (3.5) is taken into account, (3.3) and (3.4) become

$$
\begin{gathered}
P c_{z}=-a \bar{h} R^{2} \phi^{\prime}, \\
\left(c / P^{2}\right)_{u}+(h / P)_{z}=0 .
\end{gathered}
$$

The $\Phi_{00}$ component of the Einstein equation (2.4) has also a very simple form:

$$
R^{\prime \prime}+\left(\phi^{\prime}\right)^{2} R=0
$$

and the other non-trivial components of (2.4) can be summarized as

$$
\begin{gathered}
6 \Lambda=\left(H \phi^{\prime}-\phi_{u}\right) \phi^{\prime}, \\
\Phi_{11}+3 \Lambda=2 \widetilde{\kappa}^{2} e^{-2 a \phi}|c|^{2} / R^{4}, \\
\Phi_{12}=2 \widetilde{\kappa}^{2} e^{-2 a \phi} \bar{h} c / R^{3}, \\
\Phi_{22}=\left(H \phi^{\prime}-\phi_{u}\right)^{2}+2 \widetilde{\kappa}^{2} e^{-2 a \phi}|h|^{2} / R^{2},
\end{gathered}
$$

where $\Lambda, \Phi_{11}, \Phi_{12}, \Phi_{22}$ are constructed from the metric (2.25) and given in the Appendix. Finally, we have the dilaton field equation (2.3) and this reduces to 


$$
P^{2}\left[\left(R^{2} / P^{2}\right) \phi^{\prime}\right]_{u}+\left[R^{2}\left(\phi_{u}-2 H \phi^{\prime}\right)\right]^{\prime}=-4 a \tilde{\kappa}^{2} e^{-2 a \phi} c^{2} / R^{2}
$$

The problem is now to determine the six functions $(R, \phi),(P, c, h)$ and $H$ as the solutions to these equations. In this process it is useful to note two distinct symmetries of the problem. One of these is the freedom of rescaling the functions:

$$
R \rightarrow w(u) R, \quad P \rightarrow w(u) P, \quad c \rightarrow w^{2} c, \quad h \rightarrow w(u) h
$$

by an arbitrary $u$-dependent function $w(u)$. It is straightforward to check that the metric (2.25), the Maxwell field (3.5) and the field equations (3.6)(3.13) are all form-invariant under (3.14). The second symmetry concerns the coordinate gauge freedom of the metric (2.25) and the corresponding tetrad rotations of (2.24). Of particular interest is the coordinate transformation:

$$
\begin{aligned}
& u \rightarrow \tilde{u}=\int f^{-1}(u) d u, \\
& r \rightarrow \tilde{r}=f(u) r+g(u),
\end{aligned}
$$

where $f(u)$ and $g(u)$ are arbitrary functions. Under such a transformation the metric function $H$ transforms to

$$
H \rightarrow \tilde{H}=f^{2} H-(\tilde{r}-g) f_{u}-f g_{u},
$$

but the metric (2.25) preserves its form. This coordinate transformation induces on the tetrad (2.24) the null rotation:

$$
l \rightarrow \tilde{l}=f^{-1} l, \quad n \rightarrow \tilde{n}=f n, \quad \tilde{m}=m,
$$

which preserves $m$ as well as the directions of $l$ and $n$. The rescalings (3.14) and the transformations (3.15)-(3.17) turn out to be valuable tools in handling the arbitrary functions that arise through integrations. It may also be of interest to note that the coupling constant $\tilde{\kappa}$ can always be set equal to one by adding an appropriate constant to $\phi$. This is, of course, manifest in (2.1). 


\section{The Case $\phi^{\prime} \neq 0, h \neq 0$}

Consider now (3.6). Provided $\phi^{\prime} \neq 0$, this equation determines $h(u, z, \bar{z})$ in terms of the other functions and thereby reduces the unknowns by one. Since the left hand side has no $r$-dependence, it also requires, unless $h=0$, that

$$
2 a R^{2} \phi^{\prime}=-U(u)
$$

where $U(u)$ is an arbitrary function. With the above choice of the factors in (4.1) one has $\left(e^{-2 a \phi}\right)^{\prime}=U e^{-2 a \phi} / R^{2}$ and the dilaton equation (3.13) can be integrated with respect to $r$. The result is an expression for $H+r(\ln P)_{u}$. Forming $\Phi_{12}$ from this expression and comparing with the right hand side of (3.11), where now $\bar{h}=2 P c_{z} / U$, shows that (3.11) is satisfied if and only if

$$
\left(a^{2}-1\right) c c_{z}=0 .
$$

Recall that the parameter $a$ labels the different dilatonic theories and $c_{z} \neq 0$ by assumption. Hence the Robinson-Trautman solutions with $\phi^{\prime} \neq 0, h \neq 0$ exist, among all the dilatonic theories, only in string theory: $a=1$. A similar set of solutions also exists in the Einstein-Maxwell theory where $a=c_{z}=0$ and $\phi=$ const. but not in any other theory with $a \neq 1$. Having noted this interesting selection within the dilatonic theories, from now on we shall assume that

$$
a=1
$$

The other subfamilies of solutions having $h=0$ or $\phi^{\prime}=0$ which we shall later study in fact allow generalizations to arbitrary values of $a$. However, we shall not be concerned with these generalizations and concentrate on the solutions of the string theory.

After substituting $\phi^{\prime}=-U / 2 R^{2}$ and integrating once with respect to $r$, (3.8) can be written as

$$
\left[\left(R^{2}\right)^{\prime}\right]^{2}=4 s(u) R^{2}+U^{2}(u)
$$

where $s(u)$ arises as a function of integration. Depending on whether $s(u)$ can be taken to be zero or not, two subcases need to be distinguished. 
The Subcase $s(u) \neq 0$ :

When $s(u) \neq 0$ both $\phi$ and $R$ can be completely determined, all $r$ integrations can be completed and the problem reduces to the solution of two coupled partial differential equations. Starting from (4.4) and utilizing the gauge freedom (3.14)-(3.18), one finds that without any loss of generality one can set

$$
e^{-2 \phi}=b-\frac{k}{r}, \quad R=r e^{-\phi},
$$

where $b$ and $k$ are constants. After introducing two real functions $Q(u, z, \bar{z})$ and $M(u, z, \bar{z})$ which satisfy

$$
Q^{2}=k M
$$

where $Q=-2 i \tilde{\kappa} c$ and the operator

$$
\Delta=2 P^{2} \partial_{z} \partial_{\bar{z}},
$$

which is essentially the Laplacian on the $u=$ const., $r=$ const. hypersurfaces, one obtains

$$
\begin{gathered}
\Phi_{1}=\frac{i}{2 \tilde{\kappa}} \frac{Q}{R^{2}}, \quad \Phi_{2}=-\frac{i}{\tilde{\kappa}} \frac{P Q_{\bar{z}}}{k R}, \\
H=\frac{1}{2 b}\left[\Delta \ln P+k(\ln P)_{u}\right]-\frac{M}{r}-r(\ln P)_{u} .
\end{gathered}
$$

The solutions belonging to this category therefore involve two parameters $b, k$ and two independent functions $P, M$. It is possible to set $b=1$ by choosing $\phi \rightarrow 0$ as $r \rightarrow \infty$ and, of course, one can choose to work with $Q$ in place of $M$. The functions $P$ and $M$ are governed by

$$
\begin{gathered}
\Delta M+k\left[4 M(\ln P)_{u}-M_{u}\right]=\frac{P^{2}}{M} M_{z} M_{\bar{z}}, \\
\Delta \Delta(\ln P)+12 b^{2} M(\ln P)_{u}-4 b^{2} M_{u}+k^{2}\left[2\left((\ln P)_{u}\right)^{2}-(\ln P)_{u u}\right]=\frac{2 b^{2} P^{2}}{k M} M_{z} M_{\bar{z}}
\end{gathered}
$$

and it can be checked that all field equations are taken into account. Equations (4.10) and (4.11) are the stringy generalizations of the vacuum Robinson-Trautman equation. When $k=0, Q=0$ and it can also be deduced 
that $M_{z}=0$. In this limit (4.10) is trivially satisfied and (4.11) reduces to the standard form of the vacuum Robinson-Trautman equation [2] by setting $b=1$. Notice that because $Q^{2}=k M$, one can replace (4.10) with

$$
\Delta Q=k P^{2}\left(Q / P^{2}\right)_{u} .
$$

In general, the solutions under consideration are algebraically special, admit no Killing vectors and can belong to various Petrov types. Suppose we now concentrate on the solutions obeying $P_{u}=0$. Then (4.12) is a heat equation on a two dimensional surface having the metric $d s^{2}=2 d z d \bar{z} / P^{2}$ and $k$ plays the role of a diffusion constant. The Gaussian curvature of this two-surface is

$$
K=\Delta \ln P .
$$

Let us specialize further to the spaces of constant curvature and normalize $K$ to $K=0, \pm 1$. Under these assumptions

$$
P=1+\frac{1}{2} K z \bar{z}
$$

and the other metric function can be written as

$$
2 H=K-\frac{2 M}{r},
$$

where we have taken $b=1$. The simplest way to satisfy (4.10) and (4.11) is then to set $M=$ const. and clearly, if $M>0, k \geq 0$. We have now obtained three different Robinson-Trautman solutions depending on the value of $K$ and they are all of Petrov type D. The significant solution among these has $K=1$ and describes the dilatonic black holes [6] if $Q^{2} \leq 2 M^{2}$.

Another solution which belongs to this subcase and which is also of Petrov type $\mathrm{D}$ is the dilatonic C-metric [12]. In this particular example both $P$ and $Q$ depend solely on a function $x=x(u, z, \bar{z})$ which is defined implicitly by

$$
G^{-1}(x) d x=\frac{1}{2}(d z+d \bar{z})+A d u,
$$

where

$$
G(x)=1-x^{2}-A r_{+} x^{3}
$$


and $A, r_{+}$are real parameters. After choosing $b=1$ and introducing

$$
\tilde{F}(x)=1+k A x
$$

one has

$$
P^{2}=2 \tilde{F}(x) / G(x), \quad Q=-q \tilde{\kappa} \tilde{F}(x),
$$

where $q$ is the fourth parameter appearing in the solution. The metric function $H$ is now expressible as

$$
H=\frac{r^{2} A^{2}}{2 \tilde{F}(x)}\left[e^{-2 \phi} G(x)-G\left(x e^{-2 \phi}-\frac{1}{A r}\right)\right] .
$$

The Subcase $s(u)=0$ :

Starting from (4.4) one can determine the $r$-dependence of all the fields completely also when $s(u)=0$ but now a different structure emerges. This time the gauge freedom (3.14)-(3.17) allows one to set

$$
\phi=\phi_{0}(u)-\frac{\epsilon}{2} \ln r, \quad R^{2}=r
$$

where $\phi_{0}(u)$ is arbitrary, $\epsilon= \pm 1$ and the coordinate $r$ is restricted to the range $r>0$. Introducing again two functions $Q(u, z, \bar{z})$ and $M(u, z, \bar{z})$ one obtains

$$
\Phi_{1}=\frac{i}{2 \tilde{\kappa}} \frac{Q}{R^{2}}, \quad \Phi_{2}=\frac{-i}{\tilde{\kappa}} \frac{Q_{\bar{z}}}{R}
$$

and

$$
H=M+Q^{2} e^{-2 \phi_{0}} r^{\epsilon}-r\left[(\ln P)_{u}+\epsilon \dot{\phi}_{0}\right],
$$

where $\dot{\phi}_{0}=d \phi_{0} / d u$. The field equations no longer impose a relationship between $Q$ and $M$ and reduce to the following:

$$
\begin{gathered}
\Delta Q-\epsilon P^{2}\left(Q / P^{2}\right)_{u}=0, \\
\Delta M+P^{2}\left(M / P^{2}\right)_{u}-2 \epsilon \dot{\phi}_{0} M=0, \\
\Delta \ln P-(\ln P)_{u}+\epsilon \dot{\phi}_{0}=(1+\epsilon) Q^{2} e^{-2 \phi_{0}},
\end{gathered}
$$

where the operator $\Delta$ is again defined by (4.7).

When all fields are assumed to have no u-dependence, (4.24)-(4.26) take a particularly simple form and become equations on the two-dimensional 
spacelike surface whose Gaussian curvature is given by (4.13). According to (4.26), the Gaussian curvature is now equal to

$$
K=(1+\epsilon) Q^{2} e^{-2 \phi_{0}}
$$

and the surface in question is locally flat if $\epsilon=-1$. For both values of $\epsilon$, $M$ and $Q$ are two independent harmonic functions on this two-surface and moreover, $K \geq 0$. Specializing to the spaces of constant curvature, if $K=0$, either $\epsilon=-1$ or $\epsilon=1, Q=0$. The two-sphere, $K=1$, is allowed only if $\epsilon=1$ in which case $2 Q^{2}=e^{2 \phi_{0}}$ and $M$ must also be constant. Note that whenever $s(u)=0$, the string frame line element is

$$
d s_{s}^{2}=e^{2 \phi_{0}}\left[2 r^{-\epsilon}\left(d u d r+H d u^{2}\right)-2 \frac{r^{1-\epsilon}}{P^{2}} d z d \bar{z}\right]
$$

and if the fields are independent of $u$ and $\epsilon=1$, the string metric is a direct product of two two-dimensional metrics. Choosing $K=1$ gives in particular

$$
d s_{s}^{2}=e^{2 \phi_{0}}\left[\frac{2}{r} d u d r+\left(1+\frac{2 M}{r}\right) d u^{2}-d \Omega_{2}^{2}\right],
$$

where $d \Omega_{2}^{2}$ is the line element of the unit two-sphere. The sign of the constant $M$ now determines the sign of the Gaussian curvature of the $(u, r)$-subspace. Choosing $M=0$ produces the throat solution of [14] and by letting $2 M=$ $-e^{-\phi_{0}}$ one can check that (4.29) reduces to the black hole plus infinite throat solution of [14. The third, asymptotically flat region plus infinite throat solution of [14], on the other hand, can be regained from the previous subcase $s(u) \neq 0$. Each of these three solutions is known to correspond to an exact conformal field theory [14].

\section{The Case $h=0, \phi^{\prime} \neq 0$}

When $h=0$ the two principal null directions of the Maxwell tensor coincide: $\Phi_{2}=0$. Clearly, in this case $c_{z}=0$ and (4.1) cannot be deduced from (3.6). From (3.7) and (3.11) it follows that

$$
(\ln P)_{u z}=0, \quad H_{z}^{\prime}=0 .
$$


Differentiating the dilaton equation (3.13) with respect to $z$ then gives the condition

$$
H_{z}\left(R^{2} \phi^{\prime}\right)^{\prime}=0 \text {, }
$$

which can be satisfied in two ways. If $H_{z} \neq 0$, one sees that (4.1) must hold. Hence the solutions with $H_{z} \neq 0$ are simply the $\Phi_{2}=0, Q=Q(u)$ specializations of the solutions discussed in Section IV. Note that for these special solutions (4.2) holds for all values of the parameter $a$.

The second way to fulfill (5.2) is, of course, to set $H_{z}=0$. Then (4.1) need not hold and one is dealing with a subset which contains new solutions. All of these solutions are of Petrov type D and can be represented by the fields having the form

$$
H=H(u, r), \quad P=P(z, \bar{z}), \quad \Phi_{1}=R^{-2}(u, r),
$$

modulo the gauge freedom (3.14)-(3.17). The two-dimensional $u=$ const., $r=$ const. sections of these spacetimes are again spaces of constant Gaussian curvature:

$$
\Delta \ln P=K
$$

and the functions $H(u, r), \phi(u, r), R(u, r)$ are governed by the field equations

$$
\begin{gathered}
R^{\prime \prime}+\left(\phi^{\prime}\right)^{2} R=0, \\
\left(R^{2} \phi^{\prime}\right)_{u}+\left[R^{2}\left(\phi_{u}-2 H \phi^{\prime}\right)\right]^{\prime}=-4 \widetilde{\kappa}^{2} e^{-2 \phi} / R^{2}, \\
K+\left(R^{2}\right)_{u}^{\prime}-\left[H\left(R^{2}\right)^{\prime}\right]^{\prime}=-4 \tilde{\kappa} e^{-2 \phi} / R^{2}, \\
-K+\left(R^{2} H\right)^{\prime \prime}=2 R^{2} \phi^{\prime} \phi_{u}+\left(R^{2}\right)_{u}^{\prime}+2 R^{2}(\ln R)_{u}^{\prime}+\left(R^{2}\right)^{\prime}(\ln R)_{u}, \\
H_{u} R^{\prime}+2 H R_{u}^{\prime}-H^{\prime} R_{u}-R_{u u}=\left(\phi_{u}-2 H \phi^{\prime}\right) R \phi_{u} .
\end{gathered}
$$

For the special case $K=1, H_{u}=R_{u}=\phi_{u}=0$, the solutions of these equations were obtained in [13 and it can be verified that similar sets of $u$-independent solutions also exist for $K=0$ and $K=-1$. 


\section{$6 \quad$ The Case $\phi^{\prime}=0$}

When the dilaton depends only on $u$ the dilaton equation (3.13) together with (3.8) can be used to infer

$$
\Phi_{1}=0, \quad R=R(u)
$$

and because (3.14) is a symmetry of the problem, one can simply set

$$
R=1
$$

This shows how the divergence of the null geodesic $l^{\mu}$ congruence is regulated in string theory by the dilaton field. When $\phi^{\prime}=0$, it follows from (6.2) that $\rho=0$ and one is dealing with non-diverging solutions which are analogs of Kundt's class in general relativity [2]. This is to be contrasted with Einstein-Maxwell theory where a passage from the Robinson-Trautman family to Kundt's class is not possible.

For the case $\phi^{\prime}=0$, all the field equations except (3.12) amount to

$$
\begin{gathered}
\Delta \ln P=0 \\
H=V(u, z, \bar{z})-r(\ln P)_{u} \\
\Phi_{2}=P \bar{\Sigma}(u, \bar{z})
\end{gathered}
$$

The general solution of (6.3) is well-known:

$$
\ln P=f(u, z)+\bar{f}(u, \bar{z})
$$

where $f(u, z)$ is an arbitrary complex function which is analytic in $z$. The function $\Sigma(u, z)$ is also analytic in $z$ but otherwise arbitrary. Since the dilaton field is not constrained by the field equations, the solutions involve three arbitrary functions $\phi(u), f(u, z)$ and $\Sigma(u, z)$. The remaining field equation (3.12) becomes

$$
\Delta V=2 \phi_{u}^{2}+4 \tilde{\kappa}^{2} e^{-2 \phi} P^{2}|\Sigma|^{2}+\left[\left(\ln P^{2}\right)_{u}\right]^{2}-\left(\ln P^{2}\right)_{u u}
$$

where the functions $\phi(u), f(u, z)$ and $\Sigma(u, z)$ act as source terms. Hence the problem is now reduced to the solution of one non-trivial differential equation $(6.7)$. 
It can be checked that such solutions belong to one of the Petrov types III, $\mathrm{N}$ or $\mathrm{O}$ :

$$
\begin{gathered}
\Psi_{2}=0 \\
\Psi_{3}=P(\ln P)_{u \bar{z}}, \\
\Psi_{4}=\left(P^{2} V_{\bar{z}}\right)_{\bar{z}}-r\left[P^{2}(\ln P)_{u \bar{z}}\right]_{\bar{z}}
\end{gathered}
$$

and the curvature scalar vanishes: $\Lambda=0$. When $\Psi_{3}=0$, one can set $P=1$ by a coordinate transformation and a redefinition of the metric function $V(u, z, \bar{z})$. In this special case the solutions are of Petrov type $\mathrm{N}$ and describe pp-waves. The pp-waves are known to be exact solutions of $\mathrm{d}=4$ string theory when $\phi(u)$ is choosen appropriately [11], [9]. If one further specializes to the conformally flat case $V_{\bar{z} \bar{z}}=0$, the solution can be brought to the form

$$
\begin{gathered}
d s^{2}=2 d u\left[d r+\left(\phi_{u}^{2}+2 \tilde{\kappa}^{2} e^{-2 \phi}\left|\Phi_{2}\right|^{2}\right) z \bar{z} d u\right]-2 d z d \bar{z}, \\
\phi=\phi(u), \quad \Phi_{2}=\Phi_{2}(u),
\end{gathered}
$$

where $\phi(u)$ and $\Phi_{2}(u)$ are arbitrary. When $\phi$ and $\Phi_{2}$ are constants such that $8 \tilde{\kappa}^{2} e^{-2 \phi}\left|\Phi_{2}\right|^{2}=1$, this particular case can be interpreted as a WZW model which is based on the six dimensional Heisenberg group [18], [8] and reduced to four spacetime dimensions.

\section{New Radiating Solutions}

Having seen all possible subfamilies of the stringy Robinson-Trautman family, we now present some new radiating solutions. The solutions that we shall consider belong to the subcase $s(u) \neq 0$ of Section IV. Before deriving these solutions it will be instructive to go to a gauge where the dilaton picks up a $u$-dependence:

$$
e^{-2 \phi}=b-\frac{D(u)}{r}, \quad R=r e^{-\phi} .
$$

This is accomplished by a coordinate transformation (3.15)-(3.17) which has a simple effect on (4.6)-(4.9). All expressions in (4.6)-(4.9) remain valid except that now

$$
Q^{2}(u, z, \bar{z})=D(u) M(u, z, \bar{z}),
$$




$$
H=\frac{1}{2 b}\left[\Delta \ln P+D(\ln P)_{u}-\dot{D}\right]-\frac{M}{r}-r(\ln P)_{u}
$$

where $\dot{D}=d D / d u$. In the new gauge the field equations (4.11) and (4.12) become

$$
\begin{gathered}
\Delta \Delta(\ln P)+12 b^{2} M(\ln P)_{u}-4 b^{2} M_{u}+D^{2}\left[2\left((\ln P)_{u}\right)^{2}-(\ln P)_{u u}\right] \\
+D \ddot{D}-3 D \dot{D}(\ln P)_{u}=\frac{2 b^{2} P^{2}}{M D} M_{z} M_{\bar{z}} \\
\Delta Q=D P^{2}\left(Q / P^{2}\right)_{u} .
\end{gathered}
$$

When $D$ is taken to be constant, $D=k$, these equations as well as (7.1)- (7.3) reduce to their previous forms. Moreover, if $D$ is any given function of $u$, one can pass to the gauge of the Section IV by the coordinate transformation

$$
\tilde{r}=\frac{k}{D(u)} r, \quad \tilde{u}=\frac{1}{k} \int D(u) d u
$$

which has a unit Jacobian. If one also introduces

$$
\begin{gathered}
\tilde{H}=\frac{k^{2}}{D^{2}} H+\frac{k \dot{D}}{D^{2}} \tilde{r} \\
\tilde{P}=\frac{k}{D} P, \quad \tilde{M}=\frac{k^{3}}{D^{3}} M, \quad \tilde{Q}=\frac{k^{2}}{D^{2}} Q
\end{gathered}
$$

then it can be checked that (4.5)-(4.12) are all valid for the tilded variables.

Suppose now $Q$ is a constant: $Q=Q_{0}$. Then $M=Q_{0}^{2} / D(u)$ can depend only on the null coordinate $u$. Let us also assume that $P=P(z, \bar{z})$ and specialize once again to the case where the Gaussian curvature $K=\Delta \ln P$ is constant. Under these assumptions,

$$
\begin{gathered}
\Phi_{1}=-\frac{i}{2 \widetilde{\kappa}} \frac{Q_{0}}{r^{2}} e^{2 \phi}, \quad \Phi_{2}=0, \\
2 H=\frac{1}{b}(K-\dot{D})-\frac{2 M}{r}, \\
d s^{2}=2 d u d r+2 H d u^{2}-r^{2} e^{-2 \phi} d \Omega_{2}^{2},
\end{gathered}
$$

where $d \Omega_{2}^{2}$ is the line element for the two-dimensional space of constant Gaussian curvature. The field equation (7.5) is then trivially satisfied and (7.4) reduces to an ordinary differential equation for $D(u)$ :

$$
D^{3} \ddot{D}+4 b^{2} Q_{0}^{2} \dot{D}=0 .
$$


Any solution of (7.12) will give us a particular member of the RobinsonTrautman family and the simplest solution is, of course, $D=k$. Choosing for convenience $b=1$, one regains in this particular case the solutions (4.15) with a constant $M$. The $K=1, \dot{D}=0$ solutions are known to possess both future and past null infinities as well as a spatial infinity and describe black holes. Let us therefore concentrate on the $K=1, \dot{D} \neq 0$ solutions which are not gauge equivalent to the black hole solutions. It is easy to see that such solutions will be still asymptotically flat in the sense that they will possess at least a portion of the future null infinity. The parameter $Q_{0}$ will be interpretable as the conserved magnetic charge of the solutions. Using the fact that the dilatonic current:

$$
\star j_{D}=\star d \phi-\tilde{\kappa}^{2} e^{-2 \phi} A \wedge \star F,
$$

where $A=A_{\mu} d x^{\mu}$, is conserved by the virtue of the field equation (2.3), one can also relate $D\left(u_{0}\right)$ to the dilatonic charge on a $u=u_{0}$ hypersurface but, of course, $D(u)$ is not conserved. The same applies to the Bondi mass $M_{B}(u)$ of the solutions which we define as

$$
M_{B}=M-\frac{1}{4 b^{2}} D \dot{D} .
$$

This definition is motivated by the field equation (7.4) and agrees with the ADM mass of the black holes at $\dot{D}=0$. If $\dot{D} \neq 0,(7.4)$ implies that

$$
\dot{M}_{B}=-(\dot{D})^{2} / 4 b^{2}
$$

and consequently, $M_{B}(u)$ is always a decreasing function of $u$. Since $D(u)$ can either increase or decrease as a function of $u$, this means that the Bondi mass can either be converted into the dilatonic charge through the gravitational radiation or both of these quantities can be radiated out to the future null infinity.

Integrating (7.12) once gives

$$
\dot{D}-2 b^{2} Q_{0}^{2} / D^{2}=C,
$$

where $C$ is a constant and depending on whether $C=0, C>0$ or $C<0$, one gets three distinct solutions. The positivity of the Bondi mass requires

$$
C D^{2} \leq 2 Q_{0}^{2} b^{2}
$$


and this condition is respected for all values of $u$ if $C \leq 0$. We shall display these solutions after choosing $b=1$. Consider first the choice $C=0$. Then

$$
D(u)=\left(c_{0}+6 Q_{0}^{2} u\right)^{1 / 3},
$$

where $c_{0}$ is an arbitrary real constant and the Bondi mass can be written as $M_{B}=M / 2$ where $M=Q_{0}^{2}\left(c_{0}+6 Q_{0}^{2} u\right)^{-1 / 3}$. For the remaining $C \neq 0$ cases, $D(u)$ involves two real constants $c_{0}, c_{1}$ and is given as an implicit function of $u$. Letting $C=2 c_{1}^{2}$, one finds that

$$
D(u)-\frac{Q_{0}}{c_{1}} \tan ^{-1}\left[\frac{c_{1}}{Q_{0}} D(u)\right]=c_{0}+2 c_{1}^{2} u,
$$

whereas for $C=-2 c_{1}^{2}$,

$$
D(u)+\frac{Q_{0}}{2 c_{1}} \ln \left|\frac{D(u)-Q_{0} / c_{1}}{D(u)+Q_{0} / c_{1}}\right|=c_{0}-2 c_{1}^{2} u .
$$

Each of these solutions is of Petrov type D. As $r \rightarrow \infty$, the only non-zero component of the Weyl spinor $\Psi_{2}$ and the curvature scalar $\Lambda$ behave as

$$
\begin{gathered}
\Psi_{2}=-(M-D \dot{D} / 6) r^{-3}+O\left(r^{-4}\right), \\
-\left(\Psi_{2}+2 \Lambda\right)=(M-D \dot{D} / 4) r^{-3}+O\left(r^{-4}\right),
\end{gathered}
$$

and (7.22) can be used to check that (7.14) agrees with the general definition of the Bondi mass given e.g. in [19].

Using (3.15)-(3.17) it is, of course, possible to represent these solutions in alternative gauges where $M$ is constant but $D$ and $Q$ are functions of $u$ or where $D$ is constant but $M$ and $Q$ are functions of $u$. There is also a gauge in which (7.19) and (7.20) can be written in a unified manner as an explicit function of $u$. This occurs when one sets $\hat{M}(\hat{u})=\lambda \hat{Q}(\hat{u}), \hat{D}(\hat{u})=\lambda^{-1} \hat{Q}(\hat{u})$ for some real constant $\lambda$. In this gauge the case $C=0$ corresponds to

$$
\hat{Q}(\hat{u})=4 \lambda^{3} \hat{u}+\hat{c}_{0}
$$

where $\hat{c}_{0}$ is constant and if $C \neq 0$, one obtains

$$
\hat{Q}(\hat{u})=\hat{c}_{0} e^{\hat{C} \hat{u}}-4 \lambda^{3} / \hat{C},
$$


where $\hat{C}=2 \lambda C / Q_{0}$. Since $M$ and $D$ scale differently under (7.6), it is manifest in this gauge that none of these solutions is gauge equivalent to the stringy black holes.

Among these solutions (7.20) is particularly interesting because, in this case the integration constants can be arranged in such a way that the solution approaches to the stringy black hole solutions at late retarded times. This property can be easily seen also in the gauge of (7.24) by setting $M_{0}=\hat{M}(\hat{u}=$ $\infty)$ and $Q_{0}=\hat{Q}(\hat{u}=\infty)$ which implies that $\lambda=M_{0} / Q_{0}$ and $\hat{C}=-4 M_{0}^{3} / Q_{0}^{4}$. Let $M_{0}>0$ which will be consistent with the fact that $M_{B} \rightarrow M_{0}$ as $\hat{u} \rightarrow \infty$. Then (7.24) gives

$$
\begin{gathered}
\hat{M}(\hat{u})=M_{0}+\left(\hat{c}_{0} M_{0} / Q_{0}\right) \exp \left(-4 M_{0}^{3} \hat{u} / Q_{0}^{4}\right), \\
\hat{Q}(\hat{u})=\left(Q_{0} / M_{0}\right) \hat{M}(\hat{u}) \\
\hat{D}(\hat{u})=\left(Q_{0}^{2} / M_{0}^{2}\right) \hat{M}(\hat{u}) .
\end{gathered}
$$

In this coordinate system the Einstein frame metric is

$$
d s^{2}=2 d \hat{u} d \hat{r}+2 \hat{H} d \hat{u}^{2}-\left(Q_{0} / \hat{Q}\right) \hat{r}^{2} e^{-2 \phi} d \Omega_{2}^{2},
$$

where $2 \hat{H}=F(\hat{u}, \hat{r})+1-2 M_{0} / \hat{r}$ and

$$
F(\hat{u}, \hat{r})=\hat{Q} / Q_{0}-1-\hat{r} \dot{\hat{Q}} / \hat{Q}-Q_{0} \dot{\hat{Q}} / 2 M_{0}-2\left(\hat{M}-M_{0}\right) / \hat{r}
$$

with $\dot{\hat{Q}}=d \hat{Q} / d \hat{u}$. The other fields are given by

$$
e^{-2 \phi}=1-\hat{D}(\hat{u}) / \hat{r}, \quad \Phi_{1}=-i e^{2 \phi} \hat{Q}(\hat{u}) / 2 \tilde{\kappa} \hat{r}^{2}, \quad \Phi_{2}=0 .
$$

After introducing the Kruskal coordinates $\hat{U}, \hat{V}$ :

$$
\begin{gathered}
\hat{U}=-e^{-\hat{u} / 4 M_{0}} \\
\hat{V}=e^{\hat{v} / 4 M_{0}}
\end{gathered}
$$

where $\hat{v}=\hat{u}+2 \hat{r}+4 M_{0} \ln \left(\hat{r} / 2 M_{0}-1\right)$, one finds that

$$
d s^{2}=\frac{32 M_{0}^{3}}{\hat{r}} e^{-\hat{r} / 2 M_{0}} d \hat{U} d \hat{V}+16 M_{0}^{2} e^{\hat{u} / 2 M_{0}} F(\hat{u}, \hat{r}) d \hat{U}^{2}-\left(Q_{0} / \hat{Q}\right) \hat{r}^{2} e^{-2 \phi} d \Omega_{2}^{2}
$$




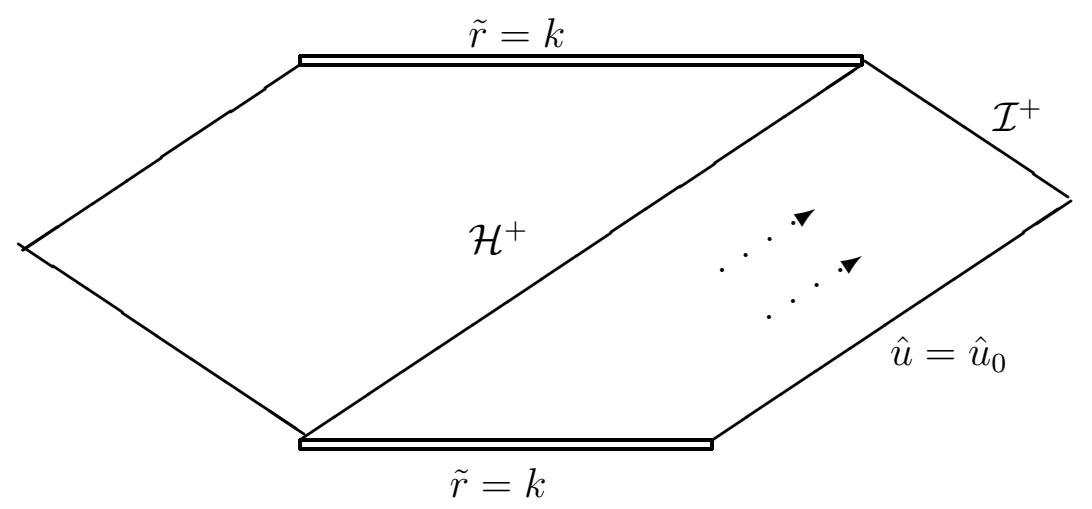

Figure 1: The Penrose diagram for the radiating solution which settles down to a stringy black hole when $Q_{0}^{2}<2 M_{0}^{2}$. The singularities are located at $\tilde{r}=k$, where $\tilde{r}$ is the radial coordinate of Section 4 . The $\hat{u} \geq \hat{u}_{0}$ portion of the future null infinity is $\mathcal{I}^{+}$and $\mathcal{H}^{+}$is the future horizon.

Since $F(\hat{u}, \hat{r}) \sim e^{-4 M_{0}^{3} \hat{u} / Q_{0}^{4}}$ as $\hat{u} \rightarrow \infty$, the solution converges exponentially fast to a solution having $F(\hat{u}, \hat{r})=0$, if $Q_{0}^{2}<2 \sqrt{2} M_{0}^{2}$. For the stringy black holes $Q_{0}^{2} \leq 2 M_{0}^{2}$ and this condition is always obeyed. Therefore, by choosing $Q_{0}^{2} \leq 2 M_{0}^{2}$, the solution can be joined smoothly to a black hole solution of mass $M_{0}$ and magnetic charge $Q_{0}$ along the null hypersurface $\mathcal{H}^{+}$which is defined by $\hat{u}=\infty$. The Penrose diagram describing the Kruskal extension of this solution for $Q_{0}^{2}<2 M_{0}^{2}$ is shown in Fig.1. Notice that, in contrast to the generic behavior of the vacuum Robinson-Trautman solutions, the extension of the present solution through the horizon $\mathcal{H}^{+}$is infinitely differentiable.

\section{Conclusions}

In this paper we have studied a class of algebraically special solutions of the low energy string theory in four dimensions under the assumption that the spacetime admits a geodesic, shear-free, non-twisting null congruence. We have called all the solutions which follow from these assumptions the stringy Robinson-Trautman family and noted that the family possesses various remarkable properties. We have seen in particular that the family preserves its identity under the transformations between the string and the Einstein frames and the Lorentz Chern-Simons three-form was found to be 
always closed. In the detailed structure of the Robinson-Trautman family the crucial role was played by the dilaton field. This massless mode of the string theory regulated the divergence of the null geodesics and together with the spin-1 field gave rise to three subclasses of solutions. Two of these subclasses were seen to be diverging while the third one had a vanishing divergence. Due to this behavior, it was possible to recover a significant number of the well-known solutions, including the stringy black holes and the pp-waves as particular members of the Robinson-Trautman family. We have also obtained some new solutions explicitly and described how a radiating solution tends smoothly to the stringy black holes at late retarded times.

Among the solutions we have studied the closest analogs of the vacuum Robinson-Trautman solutions are the ones which belong to the first subclass of Section IV. The field equations (4.10) and (4.11) which govern this subclass reduce to the vacuum Robinson-Trautman equation when $k=Q=0$. The vacuum Robinson-Trautman equation is known to be a special case of the Calabi equation 20] which arises in the study of the extremal Kähler metrics and it will be interesting to see whether a geometric interpretation can also be given for the stringy Robinson-Trautman equations (4.10) and (4.11).

In Einstein's theory the global existence and the convergence of the solutions of the vacuum Robinson-Trautman equation have been extensively studied [21. These studies have shown that the vacuum Robinson-Trautman spacetimes exist for all positive retarded times and converge exponentially fast to the Schwarzschild manifold. The solutions, however, can be extended across the event horizon only with a finite degree of smoothness. How these properties are generalized in string theory and under what initial conditions the solutions converge to the stringy black holes is an interesting open problem. The appropriate framework for this problem is again furnished by the first subclass of Section IV. In this subclass a partial answer to the question of convergence of the solutions can be given easily by specializing to the case $P=P(z, \bar{z})$. Under this assumption the parabolic equation (7.5) can be used to infer that all solutions converge to the $Q=$ const. solutions. We have examined the $Q=$ const. solutions whose Gaussian curvature is also constant in Section VII and found that there are three types of radiating solutions for the topology of a two-sphere. Only one of these solutions was seen to converge exponentially fast to the stringy black hole spacetimes and for this particular example the extension across the horizon was infinitely differentiable. Whether this is a generic behavior of all the solutions which 
belong to the first subclass of Section IV is not known at present and should be further investigated.

The radiating solution which converges to the $Q_{0}^{2}=2 M_{0}^{2}$ stringy black holes appears to be particularly interesting because both the electric [7] and the magnetic 22 extreme black holes can be interpreted as exact solutions of string theory and are supersymmetric 23]. This raises the question whether the corresponding radiating solution is also exact and supersymmetric. Since a time-like Killing vector is not present, the radiating solution cannot be supersymmetric except at $\hat{u}=\infty$. The issue of exactness, however, is more subtle and deserves further attention.

The principal goal of the present paper was to see how certain classical predictions of Einstein's theory are generalized in string theory. With all the above issues in hand, it seems reasonable to expect that stringy RobinsonTrautman family will furnish us with further insights towards the resolution of this fundamental problem.

\section{ACKNOWLEDGEMENTS}

The authors thank International Centre for Theoretical Physics, ICTP, Trieste, for hospitality where certain portions of this work was done. The research reported in this paper has been supported in part by the Scientific and Technical Research Council of Turkey, TÜBITAK and by the Turkish Academy of Sciences, TÜBA. 


\section{A Appendix}

In this Appendix we describe the NP variables in terms of exterior differential forms and compute the NP scalars for the stringy Robinson-Trautman metric (2.25). This formalism is similar to the approaches of [24] and 25] and was utilized in 26.

Let us adopt the NP spacetime conventions and represent the null tetrad in terms of a $2 \times 2$ Hermitian matrix of one-forms $\tilde{\sigma}$ :

$$
\tilde{\sigma}=\left(\begin{array}{cc}
l & \bar{m} \\
m & n
\end{array}\right) .
$$

The effect of a Lorentz transformation on $\tilde{\sigma}$ is

$$
\tilde{\sigma} \rightarrow S \tilde{\sigma} S^{\dagger}
$$

where $S \in S L(2, C)$. Cartan's first equation of structure can be written as

$$
d \tilde{\sigma}+\tilde{\sigma} \wedge \Gamma-\Gamma^{\dagger} \wedge \tilde{\sigma}=0
$$

where

$$
\Gamma=\left(\begin{array}{cc}
\Gamma_{0} & \Gamma_{2} \\
\Gamma_{1} & -\Gamma_{0}
\end{array}\right)
$$

is the $S L(2, C)$ Lie algebra valued connection. NP spin coefficients are defined as the coefficients of the complex connection one-forms:

$$
\begin{aligned}
& \Gamma_{0}=\gamma l+\epsilon n-\alpha m-\beta \bar{m}, \\
& \Gamma_{1}=-\tau l-\kappa n+\rho m+\sigma \bar{m}, \\
& \Gamma_{2}=\nu l+\pi n-\lambda m-\mu \bar{m} .
\end{aligned}
$$

By writing (A.3) explicitly, it can be checked that

$$
\begin{aligned}
d l= & -(\epsilon+\bar{\epsilon}) l \wedge n+(\alpha+\bar{\beta}-\bar{\tau}) l \wedge m+(\bar{\alpha}+\beta-\tau) l \wedge \bar{m} \\
& -\bar{\kappa} n \wedge m-\kappa n \wedge \bar{m}+(\rho-\bar{\rho}) m \wedge \bar{m}, \\
d n= & -(\gamma+\bar{\gamma}) l \wedge n+\nu l \wedge m+\bar{\nu} l \wedge \bar{m}+(\pi-\alpha-\bar{\beta}) n \wedge m \\
& +(\bar{\pi}-\bar{\alpha}-\beta) n \wedge \bar{m}+(\mu-\bar{\mu}) m \wedge \bar{m}, \\
d m= & -(\tau+\bar{\pi}) l \wedge n+(\bar{\mu}+\gamma-\bar{\gamma}) l \wedge m+\bar{\lambda} l \wedge \bar{m} \\
& -\sigma n \wedge \bar{m}+(\epsilon-\bar{\epsilon}-\rho) n \wedge m+(\beta-\bar{\alpha}) m \wedge \bar{m},
\end{aligned}
$$


The $S L(2, C)$ Lie algebra valued curvature is defined as

$$
R=d \Gamma+\Gamma \wedge \Gamma=\left(\begin{array}{cc}
\Theta_{0} & \Theta_{2} \\
\Theta_{1} & -\Theta_{0}
\end{array}\right),
$$

where the curvature two forms

$$
\begin{aligned}
\Theta_{0} & =d \Gamma_{0}-\Gamma_{1} \wedge \Gamma_{2}, \\
\Theta_{1} & =d \Gamma_{1}-2 \Gamma_{0} \wedge \Gamma_{1}, \\
\Theta_{2} & =d \Gamma_{2}+2 \Gamma_{0} \wedge \Gamma_{2}
\end{aligned}
$$

are expanded as

$$
\begin{aligned}
\Theta_{0}= & \left(\Lambda-\Phi_{11}-\Psi_{2}\right) l \wedge n+\Psi_{3} l \wedge m+\Phi_{12} l \wedge \bar{m}-\Phi_{10} n \wedge m \\
& -\Psi_{1} n \wedge \bar{m}+\left(\Psi_{2}-\Phi_{11}-\Lambda\right) m \wedge \bar{m}, \\
\Theta_{1}= & \left(\Psi_{1}+\Phi_{01}\right) l \wedge n-\left(\Psi_{2}+2 \Lambda\right) l \wedge m-\Phi_{02} l \wedge \bar{m}+\Phi_{00} n \wedge m \\
& +\Psi_{0} n \wedge \bar{m}+\left(\Phi_{01}-\Psi_{1}\right) m \wedge \bar{m}, \\
\Theta_{2}= & -\left(\Psi_{3}+\Phi_{21}\right) l \wedge n+\Psi_{4} l \wedge m+\Phi_{22} l \wedge \bar{m} \\
& -\Phi_{20} n \wedge m-\left(\Psi_{2}+2 \Lambda\right) n \wedge \bar{m}+\left(\Psi_{3}-\Phi_{21}\right) m \wedge \bar{m} .
\end{aligned}
$$

Here $\Psi_{0}, \Psi_{1}, \Psi_{2}, \Psi_{3}, \Psi_{4}$ are the components of the Weyl spinor, $24 \Lambda$ is the curvature scalar and $\Phi$ 's represent the Ricci spinor [15].

Let us define the Hodge duals:

$$
\begin{array}{ll}
\star 1=-i l \wedge n \wedge m \wedge \bar{m}, & \\
\star l=i l \wedge m \wedge \bar{m}, & \star n=-i n \wedge m \wedge \bar{m}, \quad \star m=-i l \wedge n \wedge m, \\
\star(l \wedge n)=i m \wedge \bar{m}, & \star(m \wedge \bar{m})=i l \wedge n, \\
\star(l \wedge m)=-i l \wedge m, & \star(n \wedge \bar{m})=-i n \wedge \bar{m},
\end{array}
$$

and note that $\star \star 1=-1, \quad \star \star l=l$. In these conventions one finds that for a vacuum spacetime, $R_{\mu \nu}=0$ :

$$
\star R=-i R .
$$

Consider now the metric (2.25). When the tetrad is choosen as in (2.25), the non-zero spin coefficients are

$$
\begin{array}{ccc}
\gamma=H^{\prime} / 2, & \alpha=-P_{\bar{z}} / 2 R, & \beta=P_{z} / 2 R, \\
\rho=-R^{\prime} / R, & \nu=-P H_{\bar{z}} / R, & \mu=-\left[H R^{\prime} / R+(\ln (P / R))_{u}\right] .
\end{array}
$$


The non-zero components of the Weyl spinor are found to be

$$
\begin{aligned}
\Psi_{2} & =\frac{1}{2} H^{\prime \prime}-\frac{1}{3}\left(\frac{H R^{\prime}}{R}\right)^{\prime}-\frac{1}{6 R^{2}} \Delta \ln P+\frac{1}{3}\left(\frac{R_{u}}{R}\right)^{\prime}, \\
\Psi_{3} & =\frac{P}{2 R}\left[(\ln P)_{u \bar{z}}-H_{\bar{z}}^{\prime}+\frac{2 R^{\prime}}{R} H_{\bar{z}}\right], \\
\Psi_{4} & =\frac{1}{R^{2}}\left(P^{2} H_{\bar{z}}\right)_{\bar{z}},
\end{aligned}
$$

where $\Delta=2 P^{2} \partial_{z} \partial_{\bar{z}}$. The non-zero projections of the Ricci tensor are

$$
\begin{aligned}
\Phi_{00}= & -R^{\prime \prime} / R \\
\Phi_{12}= & -\frac{P}{2 R}\left[H_{z}^{\prime}+(\ln P)_{z u}\right], \\
\Phi_{11}-3 \Lambda= & \frac{1}{2} H^{\prime \prime}-\left(\frac{R_{u}}{R}\right)^{\prime}+\frac{1}{R}\left(H R^{\prime}\right)^{\prime}+\frac{R^{\prime}}{R}[\ln (P / R)]_{u}, \\
\Phi_{11}+3 \Lambda= & \frac{1}{2 R^{2}} \Delta \ln P+\left(\frac{R_{u}}{R}\right)^{\prime} \\
& -H\left(\frac{R^{\prime}}{R}\right)^{2}-\frac{1}{R}\left(H R^{\prime}\right)^{\prime}-\frac{2 R^{\prime}}{R}[\ln (P / R)]_{u}, \\
\Phi_{22}= & \frac{1}{2 R^{2}} \Delta H+\left(H^{\prime}-2 H R^{\prime} / R\right)[\ln (P / R)]_{u}+\frac{R^{\prime}}{R} H_{u}-H^{2} \frac{R^{\prime \prime}}{R} \\
& +2 H\left(\frac{R_{u}}{R}\right)^{\prime}+[\ln (P / R)]_{u u}-\left[(\ln (P / R))_{u}\right]^{2} .
\end{aligned}
$$




\section{References}

[1] I. Robinson and A. Trautman, Phys. Rev. Lett. 4, 431 (1960); Proc. Roy. Soc. (London) A 265, 463 (1962).

[2] D. Kramer, H. Stephani, M. MacCallum and E. Herlt, Exact Solutions of Einstein's Field Equations, (Cambridge University Press, Cambridge, 1980).

[3] See e.g. P. T. Chrusciel and D. B. Singleton, Commun.Math.Phys. 147, 137(1992); J. Bicák and J. Podolsky, Phys. Rev. D ㅌ2, 887 (1995).

[4] E. N. Glass and D. C. Robinson, J. Math. Phys. 25, 3382(1984); J. D. Finley III, "The Robinson-Trautman Type III Prolongation Structure Contains $K_{2}$ ", University of New Mexico Preprint, 1995, gr-qc/9506016.

[5] D. V. Gal'tsov and O. V. Kechkin, Phys. Rev. D50(1994).

[6] G. W. Gibbons, Nucl. Phys. B207, 337(1982); G. W. Gibbons and K. Maeda, Nucl. Phys. B298, 741(1988); D. Garfinkle, G. T. Horowitz and A. Strominger, Phys. Rev. D43, 3140(1991).

[7] G. T. Horowitz and A. A. Tseytlin, Phys. Rev. Lett. $\underline{73}$, 3351(1994).

[8] C. R. Nappi and E. Witten, Phys. Rev. Lett. 71, 3751(1993).

[9] R. Güven, Phys. Lett. B191, 275(1987); D. Amati and C. Klimcik, Phys. Lett. B219, 443(1989); G. T. Horowitz and A. Steif, Phys. Rev. Lett. $\underline{64}$, 260(1990); E. Bergsoeff, R. Kallosh and T. Ortín, Phys. Rev. D47, 5444(1993).

[10] G. T. Horowitz and A. A. Tseytlin, Phys. Rev. D51, 2896(1995).

[11] A. A. Tsyetlin, Nucl. Phys. B390, 153(1993).

[12] H. F. Dowker, J. P. Gauntlett, D. A. Kastor and J. Traschen, Phys. Rev. $\underline{\mathrm{D} 49}, 2909(1994)$.

[13] M. Rakhmanov, Phys. Rev. D50, 5155(1994).

[14] S. B. Giddings and A. Strominger, Phys. Rev. D46, 627(1992); S. B. Giddings, J. Polchinski and A. Strominger, Phys. Rev. D48, 5784(1993). 
[15] E. Newman and R. Penrose, J. Math. Phys. $\underline{3}$, 566(1962); J. Math. Phys. 4, 998(1963).

[16] J. Wainwright, Int. J. Theor. Phys. 10, 39(1974).

[17] B. A. Campbell, M. J. Duncan, N. Kaloper and K. A. Olive, Nucl. Phys. B351, 778(1991).

[18] A. A. Kehagias and P. A. A. Meesen, Phys. Lett. B331, 77(1994).

[19] G. Ludwig, Gen. Rel. and Grav. ㄱ, 293(1976).

[20] E. Calabi, in Seminar on Differential Geometry, edited by S. T. Yau (Princeton University Press, Princeton 1982); K. P. Tod, Class. Quantum Grav. 6, 1159(1989).

[21] J. Forster and E. T. Newman, J. Math. Phys. $\underline{8}$, 189(1967); B. Lukács, Z. Perjés, J. Porter and Á. Sebestyén, Gen. Rel. Grav. 16, 691(1984); B. Schmidt, Gen. Rel. Grav. 20, 65(1988); A. D. Rendall, Class. Quantum Grav. ㅁ, 1339(1988); D. B. Singleton, Class. Quantum Grav. $\underline{7}$, 1333(1990); P. T. Chruściel, Commun. Math. Phys. 137, 289(1991).

[22] R. Kallosh and T. Ortín, Phys. Rev. D50, R7123(1994).

[23] G. W. Gibbons, D. Kastor, L. A. J. London, P. K. Townsend and J. Traschen, Nucl. Phys. B416, 850(1994); K. P. Tod, Class. Quantum Grav. 12, 1801(1995).

[24] K. Bichteler, Z. Physik 178, 488(1964).

[25] M. Cohen, R. Debever and L. Defrise, J. Math. Mech. 16,761(1967).

[26] R. Güven, J. Math. Phys. 17,1315(1976); R. Güven, in: Unified Field Theories of More Than Four Dimensions, eds. V. De Sabbata and E. Schmutzer(World Scientific, Singapore 1983) p.395. 